\title{
Sobrepeso y autocuidado en estudiantes de la carrera enfermería
}

\author{
Overweight and self-care in nursing students
}

\section{Excesso de peso e autocuidado em estudantes de enfermagem}

\author{
Aida Monserrate Macías Alvia \\ aidita.macias@hotmail.com. \\ Tibisay Rincón Rios \\ tibyrin@hotmail.com \\ Joel Alejandro Sanipatin Pincay \\ joel130611@hotmail.es \\ Roberth Olmedo Zambrano Santos \\ rzambranosantos@yahoo.es
}

Recibido mayo 2018 / Revisión junio 2018 / Aceptado 1 de agosto 2018

\section{RESUMEN}

Introducción: El sobrepeso es unos de los principales problemas de salud que afecta a más de un tercio de la población mundial y que tiene una íntima relación con el manejo de autocuidado, partiendo de esta realidad la problemática de esta investigación se fundamenta en el estudio del sobrepeso. Objetivo: Determinar el sobrepeso y el nivel de autocuidado en estudiantes de la carrera de enfermería de la Universidad Estatal del Sur de Manabí. Materiales y Métodos: Es una investigación descriptiva, transversal; método hipotético-deductivo, la población de estudio fue de 313 estudiantes, la muestra 187 estudiantes de quinto a octavo semestre de la carrera de enfermería, durante el periodo octubre 2018 - marzo 2019. El instrumento fue el test de autocuidado de Esther Gallego y un instrumento donde se exponen estilos de vida y alimentación diaria. Resultados y Discusión: La mayoría de estudiantes presenta sobrepeso debido a sus hábitos alimenticios, con predominio del género femenino, acompañado de un déficit en el nivel de autocuidado. Conclusión: Predomina un nivel de autocuidado medio, observándose que existe un porcentaje menor de un bajo autocuidado; demostrando que los estudiantes de la carrera de enfermería no son modelos en cuanto a la promoción de la salud y prevención de enfermedades.

Palabras clave: sobrepeso; enfermería; autocuidado

\begin{abstract}
Introduction: Overweight is one of the main health problems affecting more than a third of the world's population and has an intimate relationship with the management of self-care, starting from this reality the problem of this research is based on the study of overweight. Objective: To determine the overweight and level of self-care in nursing students at Southern Manabí State University. Materials and Methods: It is a descriptive, cross-cutting investigation; hypothetical-deductive method, the study population was 313 students, the sample 187 students from fifth to eighth semester of nursing career, during the period october 2018 - march 2019. The instrument was Esther Gallego's self-care test and an instrument where daily lifestyles and feeding are exhibited. Results and Discussion: Most students are overweight due to their eating habits, predominantly of the female gender, as well as accompanied by a deficit in the level of self-care. Conclusion: A level of average self-care predominates, noting that there is a lower percentage of a low self-care; showing that nursing students are not role models in health promotion and disease prevention.
\end{abstract}

Key words: overweight; nursing; self-care

AM: Universidad Estatal del Sur de Manabí. Ecuador. Instituto Tecnológico Superior "Portoviejo", Ecuador

TR: Universidad del Zulia Facultad de Medicina. Venezuela.

JS: Universidad Estatal del Sur de Manabí. Ecuador.

RZ: Universidad Estatal del Sur de Manabí. Ecuador. 
AM: Universidad Estatal del Sur de Manabí. Ecuador. Instituto Tecnológico Superior "Portoviejo", Ecuador

TR: Universidad del Zulia Facultad de Medicina. Venezuela.

JS: Universidad Estatal del Sur de Manabí. Ecuador.

RZ: Universidad Estatal del Sur de Manabí. Ecuador.

\section{RESUMO}

Introdução: O excesso de peso é um dos principais problemas de saúde que afetam mais de um terço da população mundial e tem uma relação íntima com a gestão do autocuidado, a partir dessa realidade o problema desta pesquisa é baseado no estudo do excesso de peso. Objetivo: Determinar o excesso de peso e o nível de autocuidado em estudantes de enfermagem da Universidade Estadual do Sul de Manabí. Materiais e Métodos: É uma investigação descritiva e transversal; método hipotético-dedutivo, a população do estudo foi de 313 alunos, a amostra de 187 alunos do quinto ao oitavo semestre de carreira de enfermagem, durante o período outubro de 2018 - março de 2019. O instrumento foi o teste de autocuidado de Esther Gallego e um instrumento onde estilos de vida diários e alimentação são exibidos. Resultados e Discussão: A maioria dos alunos está acima do peso devido aos seus hábitos alimentares, predominantemente do sexo feminino, acompanhado de um déficit no nível de autocuidado. Conclusão: Predomina um nível de autocuidado médio, observando que há uma porcentagem menor de um baixo autocuidado; mostrando que os estudantes de enfermagem não são exemplos na promoção da saúde e na prevenção de doenças.

Palavras-chave: sobrepeso; enfermagem; autocuidado

\section{INTRODUCCIÓN}

$\mathrm{E}$ n los últimos años se ha observado un aumento importante del sobrepeso y obesidad, llegando a ser considerado por la Organización Mundial de la Salud (OMS) la epidemia del siglo XXI, convirtiéndose en un serio problema de salud pública en el mundo que afecta tanto a países de altos ingresos como a naciones pobres o en vías de desarrollo.

Las conductas pocos saludables son las principales causas de la morbilidad y mortalidad prematura en este grupo de personas con problemas de sobrepeso y obesidad. Son cuatro las causas principales que influyen directamente en el desarrollo de sobrepeso y obesidad, enfermedades cardiovasculares, cáncer y diabetes: inactividad física, tabaquismo, consumo de alcohol y mala calidad de alimentación (1).

Según la Organización Mundial de la Salud desde 1975, el sobrepeso y la obesidad se han triplicado en todo el mundo. En el año 2016 más de 1900 millones de adultos de 18 o más años, tenían sobrepeso de los cuales más de 700 millones eran obesos, el 39\% de las personas adultas de 18 o más años tenían sobrepeso, y el 13\% eran obesas (2).

"El sobrepeso y la obesidad Según la Organización Mundial de la Salud (OMS) se definen como la acumulación excesiva de grasa que puede ser perjudicial para la salud", para medirla en adultos se emplea el índice de masa corporal (IMC) el cual se obtiene al dividir el peso de la persona en kilos entre el cuadrado de sus estatura en metro $(\mathrm{kg} / \mathrm{m} 2)$ (3). El sobrepeso en el ser humano es el que supera el valor indicado de acuerdo a la altura, se asocia a un nivel elevado de grasa, acumulación de líquido o por un desarrollo excesivo de músculos en los huesos (4). La prevalencia mundial de obesidad se ha duplicado en los últimos 35 años (5) y se estima que cada año fallecen al menos 2.8 millones de personas adultas como consecuencia del sobrepeso y la obesidad (6).

A pesar de la prevalencia de sobrepeso en el mundo y la amplia documentación existente sobre las consecuencias asociadas a hábitos poco saludables, existe evidencia que demuestra que hay una alta proporción de adultos jóvenes, en especial estudiantes universitarios, que asumen comportamiento nocivos para su salud (1). La necesidad de estudiar trae muchos tipos de factores como económicos y sociales que afectan el comportamiento relacionado con el estilo de vida con lo cual traen problemas asociado al exceso de peso. 
La Encuesta Nacional de Salud y Nutrición (ENSANUT) 2012, reporta que uno de cada cinco adolescentes de 12 a 19 años de edad, tiene sobrepeso y uno de cada diez, obesidad. El aumento de peso corporal es actualmente uno de los retos más importantes de Salud Pública en el mundo, dado su extensión, la rapidez de su incremento y efecto negativo que ejerce sobre la salud de la población que la padece, debido que amplía significativamente el riesgo de enfermedades crónicas no transmisibles (6), esto puede prevenirse con una alimentación sana y ejercicios.

Según ENSANUT en Ecuador, el 29,9\% de niños entre 5 a 11 años tienen obesidad. Este dato incrementa al $62 \%$ en adultos (de 19 a 59 años). El problema del sobrepeso y la obesidad radica en el riesgo de desarrollar enfermedades crónicas no transmisibles. De acuerdo a las cifras del Instituto Nacional de Estadísticas y Censo (INEC), estas son algunas de las principales causas de muerte en Ecuador (7), perfectamente prevenibles con conductas de autocuidado.

El autocuidado acorde con Denyes, Orem y Bekel es una conducta que existe en situaciones concretas de la vida, dirigida por una persona sobre sí misma, hacia los demás o hacia el entorno, esta conducta le permite regular los factores que afectan a su vida, salud o bienestar. En el contenido del bienestar, una persona que se cuida es responsable de su estado actual y actúa en consecuencia para mejorarlo (8). Son acciones que constituyen el propio cuidado y debe ser realizada de manera individual para mantener la salud $(9,10)$

Actualmente, el autocuidado como tratamiento en las enfermedades crónicas no transmisibles va en aumento debido a los resultados positivos para las personas que lo optan. La adopción de prácticas de autocuidado, como cuidar la alimentación y realizar ejercicio, mejora la calidad de vida de las personas y su entorno familiar y social (11).

Los estudios han demostrado que las mujeres utilizan más servicios de salud comparado a los hombres, pero subestiman su estado de salud en comparación con los hombres. No se encontró una relación significativa entre la educación y el estilo de vida, debido a la similitud del nivel educativo (12).

Dorothea Oren concibe al ser humano como un organismo biológico, racional y pensante. Los seres humanos tienen la capacitad de reflexionar sobre sí mismo y su entorno, capacidad para simbolizar lo que experimentan y usar creaciones simbólicas para comunicarse y guiar los esfuerzos para hacer cosas que son beneficiosas para sí mismo y otros. Define los cuidados de enfermería como el ayudar al individuo a recuperarse de la enfermedad y afrontar las consecuencias de ésta (13).

Según la OMS, la obesidad se puede clasificar en: Normopeso, es el índice de masa corporal ideal de una persona con peso normal con respecto a su estatura, el índice de masa corporal está entre los 18,5 - 24,9 kg/m2. Sobrepeso, se considera como el aumento de peso corporal por encima de un patrón entre el índice de más corporal entre los $25-29 \mathrm{~kg} / \mathrm{m} 2$. Obesidad grado 1, es un factor de riesgo para la aparición de ciertas enfermedades. Se puede determinar una persona obesidad grado 1, cuando su índice de masa corporal está entre los 30 - $34 \mathrm{~kg} / \mathrm{m} 2(14,15)$.

La determinación del sobrepeso y de la obesidad forma parte del conjunto de factores que constituyen el modo de vida de las poblaciones modernas que consumen cada vez más alimentos procesados, energéticamente densos y ricos en azúcares, grasas y sodio con una cantidad de calorías consumidas más allá de la necesidad individual $(16,17)$. La 
consecuencia de éstas, tiene implicaciones fundamentales a lo largo de todo el ciclo de vida; esta problemática es una enfermedad compleja, multifactorial y prevenible en gran medida que afecta, junto con el sobrepeso, a más de un tercio de la población mundial actual $(18,19)$.

El sobrepeso se ve ocasionado por aumento del consumo energético de alimentos, junto a una disminución de actividad física y el aumento de sedentarismo, lleva asociado una importante morbilidad, suponiendo el $44 \%$ de la carga de diabetes, $23 \%$ de las cardiopatías isquémicas y entre el 7 y $41 \%$ de la carga de algunos tipos de cáncer relacionado como son el cáncer de colon, de endometrio y el de mama (20).

Sobre las bases de lo expuesto, la problemática de esta investigación está dada por el sobrepeso, el objetivo fue determinar el sobrepeso y el nivel de autocuidado en estudiantes de la carrera de enfermería de la Universidad Estatal del Sur de Manabí.

\section{MATERIALES Y MÉTODOS}

I nvestigación descriptiva con un diseño de campo y transversal; el método utilizado hipotético- deductivo, el método empírico estuvo basado en la observación, apoyado en la técnica de la encuesta; el universo estuvo constituido por los 313 estudiantes. Se aplicó la técnica de cálculo de la muestra aleatorio estratificado, con un nivel de significancia de $+-5 \%$. El total de la muestra para el estudio fue de (187) estudiantes mayores de 18 años de edad, sin distingo de etnia, género o procedencia, quienes aceptaron y firmaron el consentimiento, quienes cursaban de quinto al octavo semestre de la carrera durante el periodo de octubre 2018 a marzo 2019. El instrumento aplicado fue la escala de Agencia de Auto Cuidado de Esther Gallego, para identificar el régimen alimenticio. Se utilizó el softwareSPSS.

\section{RESULTADOS}

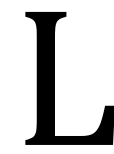
a muestra total seleccionada fue de 187 estudiantes de enfermería de la Universidad Estatal del Sur de Manabí y de acuerdo al instrumento aplicado, los resultados obtenidos se muestran a continuación:

Tabla 1. Distribución del Nivel de Agencia de autocuidado de los estudiantes de la carrera de enfermería

\begin{tabular}{llll}
\hline Orden & Alternativa & F & $\%$ \\
\hline A & Baja & 57 & $30,4 \%$ \\
B & Media & 80 & $42,6 \%$ \\
C & Alta & 50 & 27 \\
\hline Total & & 187 & 100 \\
\hline
\end{tabular}

Fuente: Instrumento aplicado 
Tabla 2. Perfil sociodemográfico del total de la población

\begin{tabular}{llll}
\hline VARIABLES & & N & $\%$ \\
\hline Edad & 20 - 30 años & 186 & 99 \\
& $31-40$ años & 1 & 1 \\
\multirow{3}{*}{ Sexo } & 41 ó más & 0 & 0 \\
& Masculino & 18 & 10 \\
Estado civil & Femenino & 168 & 90 \\
& Soltero/a & 171 & 91 \\
& Unión de hecho & 14 & 8 \\
& Casado/a & 2 & 1 \\
& Divorciado/a & 0 & 0 \\
Viudo/a & 0 & 0 \\
№ de hijos & Sin hijos & 136 & 34 \\
& 1 hijo & 35 & 19 \\
\multirow{3}{*}{ Procedencia } & 2 hijos & 15 & 8 \\
& 3 hijos o más & 1 & 1 \\
Situación académica & Urbana & 178 & 95 \\
& Rural & 9 & 5 \\
& Satisfactoria & 138 & 74 \\
& Condicional & & 26 \\
\hline
\end{tabular}

Fuente: Instrumento aplicado

Tabla 3. Índice de sobrepeso detectado en la población

\begin{tabular}{llll}
\hline \multicolumn{1}{c}{ Orden } & \multicolumn{1}{c}{ Alternativa } & F & $\%$ \\
\hline A & Bajo peso & 11 & 6 \\
B & Normal & 98 & 52 \\
C & Sobrepeso & 75 & 40 \\
D & Obesidad moderada & 75 & 2 \\
E & Obesidad Severa & - & - \\
F & Obesidad mórbida & - & - \\
\hline \multicolumn{1}{c}{ Total } & & 187 & 100 \\
\hline
\end{tabular}

Fuente: Instrumento aplicado

Tabla 4. Requerimiento alimenticio de los estudiantes de la carrera de enfermería

\begin{tabular}{llll}
\hline \multicolumn{1}{c}{ Orden } & \multicolumn{1}{c}{ Alternativa } & F & $\%$ \\
\hline A & Grasas y azúcares & 21 & 11 \\
B & Leguminosas y de origen animal & 103 & 55 \\
C & Frutas y verduras & 41 & 22 \\
D & Cereales y tubérculos & 22 & 12 \\
\hline Total & & 187 & 100 \\
\hline
\end{tabular}

Fuente: Instrumento aplicado 


\section{Antropometría}

Se calcularon el peso $(\mathrm{kg})$ y la talla (m), y se computó el índice de masa corporal $\left(\mathrm{IMC}=\right.$ peso $/$ talla $\left.^{2}, \mathrm{~kg} / \mathrm{m}^{2}\right)$. La determinación del peso se realizó con ropa muy ligera y utilizando una báscula. La talla se determinó con el sujeto descalzo y utilizando un tallímetro. Las mediciones fueron efectuadas por un único observador atendiendo a protocolos estandarizados. El IMC se interpretó utilizando la clasificación de sobrepeso y obesidad establecida. Mediante la toma de peso y talla se comprobó que los estudiantes presentan un $40 \%$ de sobrepeso, y un $2 \%$ obesidad moderada.

\section{DISCUSIÓN}

$\mathrm{E}$ l elevado porcentaje de sobrepeso encontrado difiere de los estudios donde se encontró la prevalencia de sobrepeso u obesidad en hasta $20 \%$ en la población $(21,22)$ e inferior a la reportada por Martins (23), en una universidad pública de Brasil, donde se estableció un exceso de peso del 18.2\%; $15,2 \%$ para el sobrepeso y $3.0 \%$ para obesidad.

Los jóvenes estudiados en su mayoría solteros, de 20 a 30 años y sin hijos, que viven en el área urbana, se insertan a la vida universitaria de manera independiente es un periodo crítico para el desarrollo de hábitos que se verán reflejados posteriormente en su estado de salud, comportamientos relacionados al consumo de alimentos, poca actividad física, consumo de alcohol, saltarse las comidas o no hacerlas, privilegiar la comida rápida en función de sus obligaciones. Todos ellos son considerados como factores de riesgo para el desarrollo de sobrepeso y obesidad (22).

Los estudiantes objeto de esta investigación, reportan un mayor consumo de carnes y leguminosas no obstante, existe controversia, porque no concuerdan con los datos obtenidos mediante el peso y talla, IMC, debido al elevado porcentaje de sobrepeso encontrado, lo que demuestra que existe la posibilidad que los participantes no están consumiendo alimentos saludables. La diferencia detectada podría deberse a que si bien comen proteínas probablemente sea en cocción tipo fritura, acompañada de abundantes carbohidratos, sin olvidar el consumo de azúcares que podría estar incidiendo así como también, información no declarada.

Cervera encontró en su investigación que existía un déficit en la alimentación balanceada de un grupo de estudiantes, que se reflejó en el poco o nulo consumo de frutas y verduras, donde la dieta fue de baja calidad y se caracterizó por el bajo consumo de frutas y verduras, lo que ha sido reconocido como factor de riesgo de las enfermedades cardiovasculares y algunos tipos de cáncer. No obstante, es necesario mencionar que la elección de los alimentos, su preparación y las porciones consumidas, son decisiones que dependerán de cada estudiante, pero que determinarán el estado nutricional y en el desarrollo de estilos de vida saludables que tienen importancia en su futuro (24). Es necesario recordar que estos estudiantes de la enfermería, deben ser capaces de cuidar su propia salud para poder cuidar la de otros, ser un modelo en cuanto a la práctica del autocuidado.

\section{CONCLUSIÓN}

$\mathrm{S}$ e concluye que de acuerdo a la muestra de estudiantes seleccionados para el estudio de esta investigación, predomina un Nivel de Autocuidado Medio, sin embargo, llama la atención que existe un porcentaje menor de un bajo autocuidado; lo que preocupa porque siendo carrera de salud no se deberían evidenciar estos resultados. Los profesionales de enfermería deben ser 
modelos en cuanto a la promoción de la salud y prevención de enfermedades.

Conflicto de intereses: ninguno declarado por los autores.

Fuente de financiamiento: Financiación Autofinanciado.

Agradecimientos: Ninguno declarado por los autores.

\section{REFERENCIAS}

1. González L, Carreño-Aguirre C, Estrada A, Monsalve-Álvarez J, Álvarez L. Exceso de peso corporal en estudiantes universitarios según variables sociodemográficas y estilos de vida. Rev. chil. nutr. [Internet]. 2017 [citado 2017 febrero]; 44(3): 251-261. Disponible en: https://scielo.conicyt.cl/scielo.php?script=sci ar ttext\&pid=S0717-

2. Ibáñez L. El problema de la obesidad en América. Rev. Chal. Sir. [Internet]. 2007 Dic [citado 201727 de enero]; 59(6): 399-400. Disponible en: https://scielo.conicyt.cl/scielo.php?script=sci_ar ttext\&pid=S0718-40262007000600001\&lng=es

3. Rodrigo J. Epidemiología de la obesidad mórbida. Manual de obesidad mórbida. Madrid: Médica Panamericana; 2015

4. Rina M. Comportamiento epidemiológico de la obesidad y factores de riesgo asociados en la población rural de Cumbe, Ecuador. Arch. venez. farmacol. ter. [Internet]. 2017 [citado 2017 febrero]; 36(2):88-96 - Disponible en: https://www.researchgate.net/publication/3183 49634_comportamiento_epidemiologico_de_la obesidad_y_factores_de_riesgo_asociados_e n_la_poblacion_rural_de_Cumbe_Ecuador

5. Jiménez-Talamantes $R$, Rick $J$, Quiles $J$. Diferencias entre la prevalencia de obesidad y exceso de peso estimadas con datos declarados o por medición directa en adultos de la comunidad valenciana. Nutra. Host. [Internet]. 2017 [citado 2017 febrero]; 34(1): 128-133. http://dx.doi.org/10.20960/nh.988

6. Saucedo-Molina T, Rodríguez J, Oliva L Villarreal M, León C, Fernández T. Relación entre el índice de masa corporal, la actividad física y los tiempos de comida en adolescentes mexicanos. Nutra. Host. [Internet]. 2015 [citado 2017 febrero]; 32(3): 1082 1090.http://dx.doi.org/10.3305/nh.2015.32.3.93 31

7. Córdoba D, Carmona M, Terán O, Márquez M. Relación del estilo de vida y estado de nutrición en estudiantes universitarios: estudio descriptivo de corte transversal. Medwave [Internet]. 2013 [citado 2017 febrero]; 13(11): e5864.doi: 10.5867/medwave.2013.11.5864

8. Pardo MP, Núñez NA. Estilo de vida y salud en la mujer adulta joven. Aquichan [Internet].
2008; 8(2):266-284.Disponible en: https://www.redalyc.org/pdf/741/74180

9. 212.pdfontr

10. Gallardo L. El autocuidado y las apps, agentes de cambio en enfermedades como sobrepeso, obesidad y diabetes. RDU [Internet]. 2017 [citado 2017 noviembre]; 18(8). http://doi.org/10.22201/codeic.16076079e.201 7.v18n8.a3

11. Rivera L, Díaz L. Relación entre la capacidad de agencia de autocuidado y los factores de riesgo cardiovascular. Cuad. - Hosp. Clín. [Internet]. 2007 Jul [citado 2017 enero]; 52(2): 30-38. Disponible en: http://www.scielo.org.bo/scielo.php?script=sci_ arttext\&pid=S165267762007000200005\&lng=es

12. Escobar MP, Pico ME. Autocuidado de la salud en jóvenes universitarios, Manizales, 2010-2011. Rev. Fac. Nac. Salud Pública [Internet]. 2013 [citado 2017 febrero]; 31(2): 178-186. Disponible

en: http://www.scielo.org.co/pdf/rfnsp/v31n2/v31n2 a03.pdf

13. Herranz A, López MC, San Julián C. Influencia del exceso de peso en la calidad de vida relacionada con la salud de los adolescentes. An. Pediatr. [Internet]. 2015 [citado 2017 febrero]; 82(3). doi: 10.1016/j.anpedi.2014.06.019

14. Naranjo HY, Concepción PJA, Rodríguez LM. La teoría déficit de autocuidado: Dorothea Elizabeth Orem. Gaceta médica espirituana [Internet]. 2017;19(3).Disponible en: https://www.medigraphic.com/cgibin/new/resumen.cgi?IDARTICULO=77397

15. Debayle M. Tipos de sobrepeso y sus riesgos [Internet]. 2017. Disponible en: www.revistamoi.com/salud/tipos-desobrepeso/

16. Centro Nacional para la Prevención de Enfermedades Crónicas y Promoción de la Salud, División de Nutrición. Actividad física y obesidad. Índice de masa corporal [Internet]. $2015 . \quad$ Disponible en: https://www.cdc.gov/healthyweight/spanish/ass essing/bmi/index.html

17. García-Continente X, Allúe N. Hábitos alimentarios, conductas sedentarias $y$ sobrepeso y obesidad en adolescentes de Barcelona. Sciencedirect [ Internet]. 2015 [citado 2017 junio] 83(1). Disponible en: https://www.sciencedirect.com/science/article/p ii/

18. Cavalcante J. Asociación entre exceso de peso y características de adultos jóvenes escolares: contribución para el cuidado de enfermería. Latino-Am. Enfermagem [Internet]. 2015 [citado 2017 febrero]; 23(2).Disponible en:

http://www.scielo.br/pdf/rlae/v23n2/es_01041169-rlae-23-02-00250.pdf 
19. Oliveira A, Nogueira M. Obesidade como fator de risco para a hipertensão entre profissionais de enfermagem de uma instituição filantrópica. Rev. esc. enferm. USP [Internet]. 2010 June [citado 2017 enero]; 44(2): 388394.doi.org/10.1590/S0080623420100002000 21

20. Villacreses N. Estilos de vida relacionados con sobrepeso y obesidad en los comerciantes del mercado de Jipijapa. México: Jipijapa.UNESUM; 2017. Disponible en: http://repositorio.unesum.edu.ec/handle/53000/ 910

21. Varela M, Duarte C, Salazar I, Lema L. Tamayo J. Actividad física y sedentarismo en jóvenes universitarios de Colombia: prácticas, motivos y recursos para realizarlas. Colomb med [Internet]. 2011 [citado 2017 marzo]; 42(3):269-277. Disponible en: http://www.scielo.org.co/pdf/cm/v42n3/v42n3a 2.pdf

22. Von Bothmer MI, Fridlund B. Gender differences in health habits and in motivation for a healthy lifestyle among Swedish university students. Nurs Health [Internet]. 2005 [citado 2017 marzo]; 7 (2): 107-18. Disponible

en: http://www.ncbi.nlm.nih.gov/pubmed/15877687

23. Arroyo M, Rocandio P, Ansotegui L, Pascual E. Calidad de la dieta, sobrepeso y obesidad en estudiantes universitarios. Nutr. Hosp. [Internet]. 2006 dic [citado 201727 de enero]; 21(6): 673-679. Disponible en: http://scielo.isciii.es/scielo.php?script=sci_artte xt\&pid=S0212-16112006000900007\&lng=es

24. Martins M, Ricarte I, Rocha C. Blood pressure, excess weight and level of physical activity in students of a public university. Arq. Bras. Cardiol. [Internet]. 2010 [citado 2017 de enero]; 95(2): 192-199.doi.org/10.1590/S0066782X2010005000069

25. Rodríguez R, Palma L, Ximena R, Escobar B, et al. Hábitos alimentarios, actividad física y nivel socioeconómico en estudiantes universitarios de Chile. Nutr. Hosp. [Internet]. $2013 \mathrm{abr}$ [citado 201727 de enero]; 28(2): 447-455.doi.org/10.3305/nh.2013.28.2.6230 